\title{
Quasiparticles as detector of topological quantum phase transitions
}

\author{
Sourav Manna, ${ }^{1}$ N. S. Srivatsa $\odot,{ }^{1}$ Julia Wildeboer $\odot,{ }^{1,2,3}$ and Anne E. B. Nielsen ${ }^{1, *}$ \\ ${ }^{1}$ Max-Planck-Institut für Physik komplexer Systeme, D-01187 Dresden, Germany \\ ${ }^{2}$ Department of Physics, Arizona State University, Tempe, Arizona 85287, USA \\ ${ }^{3}$ Department of Physics and Astronomy, University of Kentucky, 505 Rose Street, Lexington, Kentucky 40506, USA
}

(Received 17 January 2020; revised 16 November 2020; accepted 15 December 2020; published 31 December 2020)

\begin{abstract}
A number of tools have been developed to detect topological phase transitions in strongly correlated quantum systems. They apply under different conditions, but do not cover the full range of many-body models. It is hence desirable to further expand the toolbox. Here, we propose to use quasiparticle properties to detect quantum phase transitions. The approach is independent from the choice of boundary conditions, and it does not assume a particular lattice structure. The probe is hence suitable for, e.g., fractals and quasicrystals. The method requires that one can reliably create quasiparticles in the considered systems. In the simplest cases, this can be done by a pinning potential, while it is less straightforward in more complicated systems. We apply the method to several rather different examples, including one that cannot be handled by the commonly used probes, and in all the cases we find that the numerical costs are low. This is so, because a simple property, such as the charge of the anyons, is sufficient to detect the phase transition point. For some of the examples, this allows us to study larger systems and/or further parameter values compared to previous studies.
\end{abstract}

DOI: 10.1103/PhysRevResearch.2.043443

\section{INTRODUCTION}

Describing physical systems in terms of phases allows us to focus on key properties rather than the full set of microscopic details. Quantum phase transitions take place at zero temperature, when a control parameter, such as the magnetic field strength, is varied [1]. In conventionally ordered phases, quantum phase transitions can be characterized by a local order parameter, arising from the broken symmetry of the system, but this approach breaks down for the case of topologically ordered systems [2]. A further complication arises because strongly correlated quantum many-body systems are demanding to study numerically. Density matrix renormalization group investigations are usually limited to one-dimensional systems or quasi-two-dimensional systems such as ladders and thin cylinders [3], and many systems that may harbor topologically ordered phases cannot be studied with large-scale quantum Monte Carlo, due to the sign problem [4].

Different probes have been developed to detect topological phase transitions, such as ground state degeneracy [5], manybody Chern number [6-8], spectral flow [9-11], entanglement spectrum [11-14], topological entanglement entropy [15-17],

* On leave from Department of Physics and Astronomy, Aarhus University, DK-8000 Aarhus C, Denmark.

Published by the American Physical Society under the terms of the Creative Commons Attribution 4.0 International license. Further distribution of this work must maintain attribution to the author(s) and the published article's title, journal citation, and DOI. Open access publication funded by the Max Planck Society. and fidelity [18]. The first three assume particular boundary conditions. The entanglement-based probes have been tested for regular structures in two dimensions, but it is not clear how and whether they can be applied in highly irregular systems. Fidelity cannot be used if the Hilbert space itself changes as a function of the parameter. There are hence systems that cannot be handled currently. In addition, it is desirable to find probes that are less costly numerically. There is hence a strong demand for identifying further probes.

Here, we show that quasiparticles are an interesting tool to detect topological quantum phase transitions. It is well known that topologically ordered systems can host anyons, and their properties define the topological phase. Anyons are quasiparticles that are neither fermions nor bosons, and this can be seen from the braiding statistics. They can also have fractional charge. Both anyonic braiding properties and fractional charge have been confirmed in numerical studies [19-23]. Here, we propose to use quasiparticles to detect phase transitions that happen when a parameter in the Hamiltonian is varied. Our starting point is to modify the Hamiltonian locally to generate quasiparticles at well-defined positions in the ground state. In the simplest case, this can be done by adding a potential, while in more complicated systems, it may require some ingenuity [24]. We then study the properties of the quasiparticles as a function of the parameter. When the two phases do not support the same set of quasiparticles, a change is seen at the phase transition. The method can be applied for all types of anyons, as long as there is an appropriate way to create the anyons, and it does not require a particular choice of boundary conditions or a particular lattice structure. The method therefore also applies to, e.g., disordered systems, fractals, and quasicrystals.

We test the method on concrete examples, namely phase transitions happening in a lattice Moore-Read model on 
a square lattice and on a fractal lattice, in an interacting Hofstadter model in the presence and in the absence of disorder, and in Kitaev's toric code in a magnetic field. Among these models, we include cases for which the phase transition point is already known, since this allows us to compare with other methods and check the reliability of the anyon approach. For all these examples, we find that it is sufficient to compute a relatively simple property, such as the charge of the anyons, to determine the phase transition point. The computations can therefore be done at low numerical costs.

For the Moore-Read model on a square lattice, e.g., a large speedup is found compared to previous computations of the topological entanglement entropy, and this enables us to determine the transition point much more accurately. For the interacting Hofstadter model, we only need two exact diagonalizations for each data point, which is much less than what is needed to compute the many-body Chern number. Finally, for the model on the fractal, we do not know of other methods that could be used for detecting the phase transition.

\section{LATTICE MOORE-READ MODEL}

The Moore-Read state is a trial wave function to describe the plateau at filling factor $5 / 2$ in the fractional quantum Hall effect [25], and it supports non-Abelian Ising anyons [20]. In this section, we investigate phase transitions that happen in lattice versions of the Moore-Read state on two different lattices as a function of the lattice filling.

\section{A. Moore-Read model on a square lattice}

We investigate a model with a particular type of lattice Moore-Read ground state, which was shown in Ref. [26], based on computations of the topological entanglement entropy $\gamma$, to exhibit a phase transition as a function of the lattice filling with the transition point in the interval $[1 / 8,1 / 2]$. A more precise value was not determined because $\gamma$ is expensive to compute numerically, since it involves computing several entanglement entropies, and these are obtained using the replica trick, which means that one works with a system size that is twice as big as the physical system. In fact, for many systems, it is only possible to compute $\gamma$ for a range of system sizes that are too small to allow for an extrapolation to the thermodynamic limit. Here, we show that the transition point can be found by computing the charge of the anyons. This quantity can be expressed as a classical mean value and is much less expensive to compute. As a result, we can determine the transition point more accurately.

We consider a square lattice with a roughly circular boundary to mimic a quantum Hall droplet. The $N$ lattice sites are at the positions $z_{1}, \ldots, z_{N}$, and the local basis on site $j$ is $\left|n_{j}\right\rangle$, where $n_{j} \in\{0,1,2\}$ is the number of bosons on the site. The lattice Moore-Read state $\left|\Psi_{0}\right\rangle$ is defined as [26]

$$
\begin{aligned}
\left|\Psi_{x}\right\rangle & \propto \sum_{n_{1}, \ldots, n_{N}} \Psi_{x}\left(n_{1}, \ldots, n_{N}\right)\left|n_{1}, \ldots, n_{N}\right\rangle, \\
\Psi_{x}\left(n_{1}, \ldots, n_{N}\right) & =\mathcal{G}_{n}^{x} \delta_{n} \prod_{i<j}\left(z_{i}-z_{j}\right)^{2 n_{i} n_{j}} \prod_{i \neq j}\left(z_{i}-z_{j}\right)^{-\eta n_{i}},
\end{aligned}
$$

We next consider the Moore-Read model on a fractal lattice. The fractal lattice is not periodic, and we can therefore not apply methods, such as ground state degeneracy, spectral flow, or many-body Chern number computations to detect a possible phase transition. Methods based on entanglement 

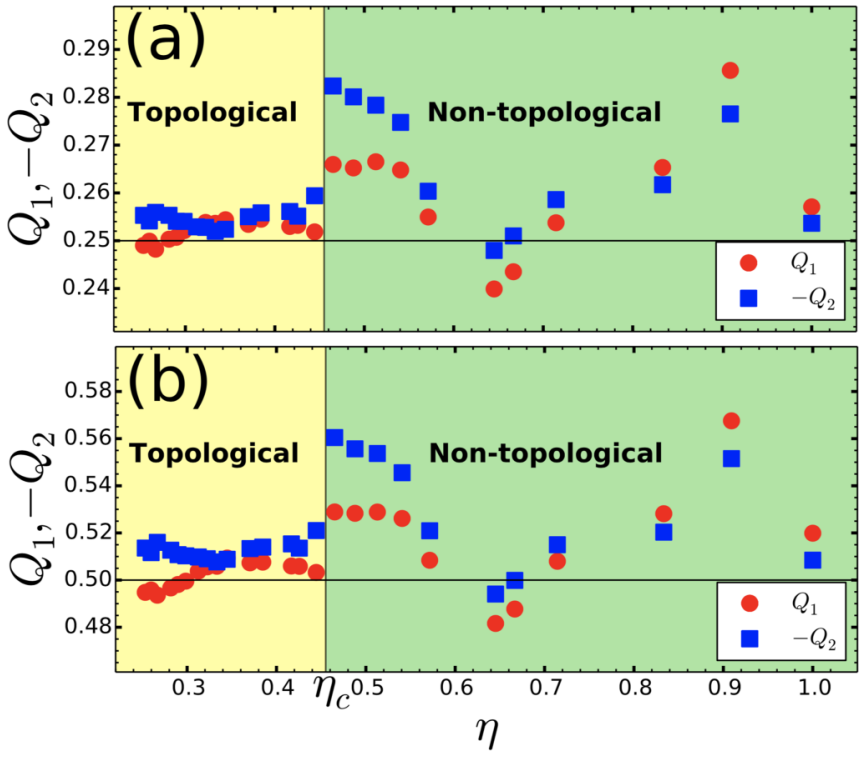

FIG. 1. (a) Excess charges $Q_{1}$ and $Q_{2}$ for the Moore-Read state $\left|\Psi_{a}\right\rangle$ on a square lattice as a function of the flux per site $\eta$. In the topological phase, $Q_{1}$ and $-Q_{2}$ are close to the charge of the positive anyon (horizontal line at $1 / 4$ ). In the nontopological phase, $Q_{1}$ and $Q_{2}$ may take any value. The jump away from $1 / 4$ predicts the transition point $\eta_{c} \in[0.44,0.46]$. (b) To test the robustness of the method, we observe that $\left|\Psi_{b}\right\rangle$ gives the same transition point. The Monte Carlo errors are of order $10^{-4}$.

computations also do not apply, since we do not have a thorough understanding of entanglement properties of topological many-body states on fractal lattices. Fidelity cannot be used either, since the Hilbert space changes when the considered parameter changes. Quasiparticle properties, on the contrary, can detect a transition, as we will now show.

Lattice Laughlin fractional quantum Hall models were recently constructed on fractals [27], and we here consider a similar construction for the Moore-Read state. Specifically, we define the state $\left|\Psi_{a}\right\rangle$ on a lattice constructed from the Sierpiński gasket with $N=243$ triangles by placing one lattice site on the center of each triangle. In Fig. 2, we vary the particle number $M \in[24,96]$ to have different $\eta \in$ $[0.19,0.79]$ values and plot the excess charges as a function of $\eta$. The excess charges are $Q_{1} \approx-Q_{2} \approx 1 / 4$ for $\eta<\eta_{c}$ and fluctuate for $\eta>\eta_{c}$, which reveals a phase transition at the transition point $\eta_{c} \in[0.43,0.46]$.

\section{DISORDERED INTERACTING HOFSTADTER MODEL}

As another example, we study a Hofstadter model for hard-core bosons on a square lattice. The clean model is known to host a topological phase for low enough lattice filling factor [28,29], and here we investigate the effect of adding a disordered potential. The system sizes that can be reached with exact diagonalization are too small to allow for a computation of the topological entanglement entropy. Instead, we use the anyon charges to show that the system undergoes a phase transition as a function of the disorder strength. This gives a large speedup in computation time compared to the many-body Chern number computations that were done in

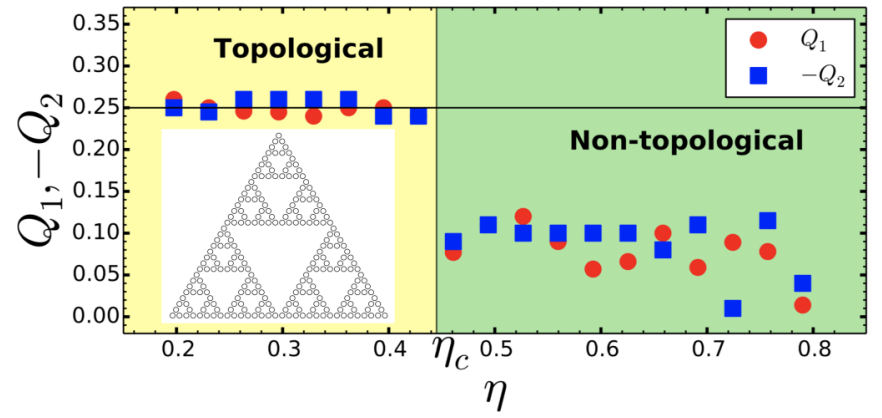

FIG. 2. Excess charges $Q_{1}$ and $Q_{2}$ for the Moore-Read state $\left|\Psi_{a}\right\rangle$ on a fractal lattice (inset) as a function of the flux per site $\eta$. In the topological phase, $Q_{1}$ and $-Q_{2}$ are close to the charge of the positive anyon (horizontal line at $1 / 4$ ). In the nontopological phase, $Q_{1}$ and $Q_{2}$ may take any value. The jump away from $1 / 4$ predicts the transition point $\eta_{c} \in[0.43,0.46]$. The Monte Carlo errors are of order $10^{-4}$.

Ref. [28]. This is so, because it only takes two exact diagonalizations per data point to get the anyon charges, while the Chern number computation involves a large number of exact diagonalizations per data point, corresponding to a grid of twist angles in two dimensions.

The Hofstadter model describes particles hopping on a two-dimensional square lattice in the presence of a magnetic field perpendicular to the plane. Hopping is allowed between nearest-neighbor sites, and the magnetic field is taken into account by making the hopping amplitudes complex. Whenever a particle hops around a closed loop, the wave function acquires a phase, which is equal to the Aharonov-Bohm phase for a charged particle encircling the same amount of magnetic flux.

We take open boundary conditions and add interactions by considering hard-core bosons. For a lattice with $N=L_{x} \times L_{y}$ sites, the Hamiltonian takes the form

$$
\begin{aligned}
H_{0}= & -\sum_{x=1}^{L_{x}-1} \sum_{y=1}^{L_{y}}\left(c_{x+1, y}^{\dagger} c_{x, y} e^{-i \pi \alpha y}+\text { H.c. }\right) \\
& -\sum_{x=1}^{L_{x}} \sum_{y=1}^{L_{y}-1}\left(c_{x, y+1}^{\dagger} c_{x, y} e^{i \pi \alpha x}+\text { H.c. }\right)+\sum_{x=1}^{L_{x}} \sum_{y=1}^{L_{y}} h_{x, y} n_{x, y},
\end{aligned}
$$

where $c_{x, y}$ is the hard-core boson annihilation operator and $n_{x, y}=c_{x, y}^{\dagger} c_{x, y}$ is the number operator acting on the lattice site at the position $(x, y)$ with $x \in\left\{1, \ldots, L_{x}\right\}$ and $y \in\left\{1, \ldots, L_{y}\right\}$. If a particle hops around a plaquette, the phase acquired is $2 \pi \alpha$, so $\alpha$ is the flux through the plaquette. We here consider the case, where the number of flux units per particle is two, i.e., $N \alpha / M=2$. The last term in (6) is the disordered potential, and $h_{x, y} \in[-h, h]$ is drawn from a uniform distribution of width $2 h$, where $h$ is the disorder strength.

In the clean model, it is well known that one can trap anyons in the ground state by adding a local potential with a strength that is sufficiently large compared to the hopping amplitude [30-32]. We here choose

$$
H_{V}=V n_{x_{1}, y_{1}}-V n_{x_{2}, y_{2}}, \quad\left(x_{1}, y_{1}\right) \neq\left(x_{2}, y_{2}\right),
$$




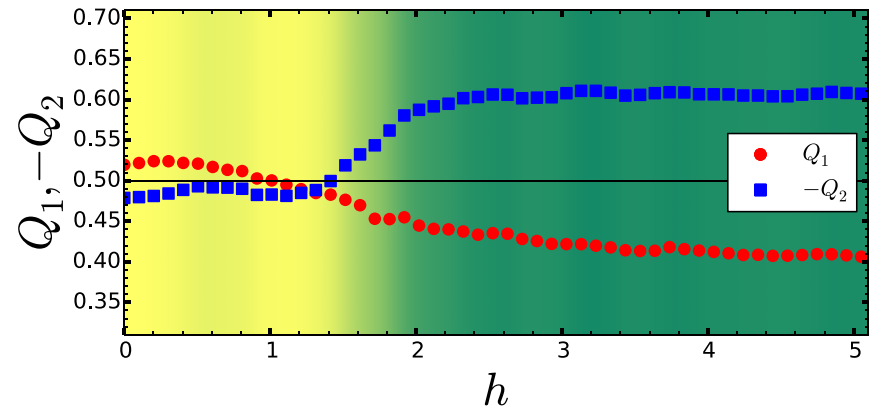

FIG. 3. Excess charges $Q_{1}$ and $Q_{2}$ as a function of the disorder strength $h$ for the interacting Hofstadter model with $M=3, N=24$, and $\alpha=0.25$. In the topological phase, $Q_{1} \approx-Q_{2} \approx 1 / 2$ (horizontal line), and the observed change away from this value predicts the transition point $h_{c} \simeq 1.5$. We average over 2000 statistically independent disorder realizations for each $h$ to ensure convergence of the data.

where $V \gg 1$. This potential traps one positively (negatively) charged anyon at the site $\left(x_{1}, y_{1}\right)\left[\left(x_{2}, y_{2}\right)\right]$.

We use the excess charge in a region around the sites $\left(x_{1}, y_{1}\right)$ and $\left(x_{2}, y_{2}\right)$ to detect the phase transition. We define the density profile as

$$
\rho(x+i y)=\left\langle n_{x, y}\right\rangle_{H_{0}+H_{V}}-\left\langle n_{x, y}\right\rangle_{H_{0}},
$$

where $\left\langle n_{x, y}\right\rangle_{H_{0}+H_{V}}$ is the particle density, when the trapping potential is present, and $\left\langle n_{x, y}\right\rangle_{H_{0}}$ is the particle density, when the trapping potential is absent. The excess charge is then defined as in (4) with $w_{1}=x_{1}+i y_{1}$ and $w_{2}=x_{2}+i y_{2}$. Here, we choose $R$ such that the circular region includes all sites up to the second-nearest-neighbor sites. The absolute value of the excess charge should be close to $1 / 2$ in the topological region, while it can take any value and may vary with $h$ in the nontopological region.

We choose a point, which is deep in the topological phase for $h=0$, namely $M=3, N=24$, and $\alpha=0.25$, and plot the excess charges as a function of the disorder strength $h$ in Fig. 3. We observe that $Q_{1}$ and $-Q_{2}$ are close to $1 / 2$ up to $h \simeq 1.5$, while the excess charges deviate more from $1 / 2$ for $h>1.5$. The data hence predict the phase transition to happen at $h_{c} \simeq 1.5$.

We can also put the disorder strength to $h=0$ and study the clean model as a function of the magnetic flux $\alpha$. Specifically, we vary $\alpha$ and the lattice filling $M / N$, while keeping the flux per particle $N \alpha / M$ fixed. For this case, it was found in Ref. [28] that there is a phase transition at $\alpha_{c} \in[0.375,0.400]$. We take values of $M$ and $N$ (see Table I), which are numerically accessible for exact diagonalization, and for each choice $\alpha=2 M / N$. Figure 4 shows that $Q_{1}$ and $-Q_{2}$ are quite close to $1 / 2$ for $\alpha$ values up to 0.375 , but for higher $\alpha$ they deviate much more from $1 / 2$. The data hence predict the transition point $\alpha_{c} \in[0.375,0.389]$, which is consistent with the result in Ref. [28].

\section{TORIC CODE IN A MAGNETIC FIELD}

To test the applicability of the method outside the family of chiral fractional quantum Hall models, we next study Kitaev's toric code $[33,34]$ on a square lattice with periodic boundary
TABLE I. We show here the different choices we make for the number of particles $M$, the shapes and sizes $N=L_{x} \times L_{y}$ of the lattices, and the fluxes per plaquette $\alpha=2 M / N$. The quantity $\operatorname{dim}(\mathcal{H})$ is the dimension of the corresponding Hilbert spaces. We display the data for the absolute values of the excess charges $Q_{+}$and $Q_{-}$. There is a significant change in $Q_{+}$and $Q_{-}$, when going from $\alpha=0.375$ to $\alpha \simeq 0.389$.

\begin{tabular}{ccccccc}
\hline \hline$M$ & $N$ & $L_{x} \times L_{y}$ & $\alpha$ & $\operatorname{dim}(\mathcal{H})$ & $Q_{+}$ & $Q_{-}$ \\
\hline 2 & 24 & $6 \times 4$ & 0.167 & 276 & 0.491 & 0.507 \\
3 & 28 & $7 \times 4$ & 0.214 & 3276 & 0.476 & 0.522 \\
3 & 24 & $6 \times 4$ & 0.250 & 2024 & 0.519 & 0.478 \\
4 & 28 & $7 \times 4$ & 0.286 & 20475 & 0.521 & 0.475 \\
4 & 24 & $6 \times 4$ & 0.333 & 10626 & 0.475 & 0.520 \\
5 & 28 & $7 \times 4$ & 0.357 & 98280 & 0.465 & 0.532 \\
6 & 32 & $8 \times 4$ & 0.375 & 906192 & 0.466 & 0.533 \\
7 & 36 & $6 \times 6$ & 0.389 & 8347680 & 0.380 & 0.610 \\
5 & 25 & $5 \times 5$ & 0.400 & 53130 & 0.332 & 0.666 \\
5 & 24 & $6 \times 4$ & 0.417 & 42504 & 0.348 & 0.650 \\
6 & 28 & $7 \times 4$ & 0.429 & 376740 & 0.250 & 0.747 \\
6 & 25 & $5 \times 5$ & 0.480 & 177100 & 0.278 & 0.719 \\
7 & 28 & $7 \times 4$ & 0.500 & 1184040 & 0.284 & 0.714 \\
7 & 25 & $5 \times 5$ & 0.560 & 480700 & 0.402 & 0.595 \\
7 & 24 & $6 \times 4$ & 0.583 & 346104 & 0.440 & 0.557 \\
\hline \hline
\end{tabular}

conditions. This system exhibits a $\mathbb{Z}_{2}$ topologically ordered phase, and it is known that a sufficiently strong, uniform magnetic field drives the system into a polarized phase [35-39]. Here, we show that anyons inserted into the system are able to detect this phase transition, and the obtained transition points agree with earlier results based on perturbative, analytical calculations and tensor network studies. Our computations rely on exact diagonalization for a system with 18 spins and are hence quite fast to do numerically. We find that the anyons are significantly better at predicting the phase transition point than the energy gap closing for the same system size.

The toric code has a spin- $1 / 2$ on each of the edges of the $N_{x} \times N_{y}$ square lattice. The Hamiltonian

$$
H_{\mathrm{TC}}=-\sum_{p} B_{p}-\sum_{v} A_{v}, \quad B_{p}=\prod_{i \in p} \sigma_{i}^{z}, \quad A_{v}=\prod_{i \in v} \sigma_{i}^{x},
$$

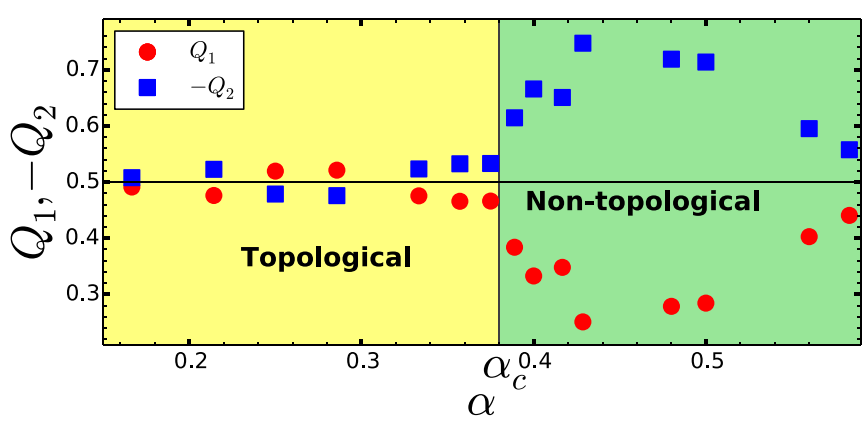

FIG. 4. Excess charges $Q_{1}$ and $Q_{2}$ as a function of the magnetic flux per plaquette $\alpha$ for the interacting Hofstadter model without disorder. In the topological phase, $Q_{1} \approx-Q_{2} \approx 1 / 2$, and the observed change away from this value predicts the transition point $\alpha_{c} \in[0.375,0.389]$. 
is expressed in terms of the Pauli operators $\sigma_{i}^{x}$ and $\sigma_{i}^{z}$, which act on the $N=2 N_{x} N_{y}$ spins. The sums are over all plaquettes $p$ and vertices $v$ of the lattice. $B_{p}$ acts on the spins on the four edges surrounding the plaquette $p$, and $A_{v}$ acts on the spins on the four edges connecting to the vertex $v$.

$H_{\mathrm{TC}}$ is exactly solvable, and the four degenerate ground states are eigenstates of $B_{p}$ and $A_{v}$ with eigenvalue 1. States containing anyons are obtained by applying certain string operators to the ground states. The string operator either changes the eigenvalue of two $A_{v}$ operators to -1 or the eigenvalue of two $B_{p}$ operators to -1 . In the former case, two electric excitations $e_{v}$ are created, and in the latter case two magnetic excitations $m_{p}$ are created. The wave function acquires a minus sign if one $m_{p}$ is moved around one $e_{v}$, and the excitations are hence Abelian anyons.

Here, we instead modify the Hamiltonian, such that anyons are present in the ground states. The ground states of the Hamiltonian $H_{m} \equiv H_{\mathrm{TC}}+2 B_{p_{1}}+2 B_{p_{2}}$ have one $m_{p}$ on each of the plaquettes $p_{1}$ and $p_{2}$. Similarly, the ground states of the Hamiltonian $H_{e} \equiv H_{\mathrm{TC}}+2 A_{v_{1}}+2 A_{v_{2}}$ have one $e_{v}$ on each of the vertices $v_{1}$ and $v_{2}$. We drive the system through a phase transition by adding a magnetic field $H_{\lambda}^{k}=\lambda \sum_{i} \sigma_{i}^{k}$ in the $k$ direction with strength $\lambda$. When $\lambda$ is large enough, it is energetically favorable to polarize all the spins, and the system is no longer topological.

Previous investigations, based on perturbative, analytical calculations and tensor network studies [35-37], have shown that $H_{\mathrm{TC}}+H_{\lambda}^{z}$ has a second-order phase transition at $\lambda_{c} \simeq$ 0.33 , while $H_{\mathrm{TC}}+H_{\lambda}^{y}$ has a first-order phase transition at $\lambda_{c}=1$. The magnetization per spin computed using exact diagonalization (Fig. 5) gives similar values for the transition points.

We now use anyons to detect the transition. We study $\left\langle A_{v_{1}}\right\rangle=\left\langle A_{v_{2}}\right\rangle$ for the Hamiltonian $H_{e}+H_{\lambda}^{k}$ and $\left\langle B_{p_{1}}\right\rangle=\left\langle B_{p_{2}}\right\rangle$ for the Hamiltonian $H_{m}+H_{\lambda}^{k} \cdot\left\langle A_{v_{1}}\right\rangle=-1$ and $\left\langle B_{p_{1}}\right\rangle=-1$ signify the presence of the anyons. In the fully polarized phase, both $\left\langle A_{v_{1}}\right\rangle$ and $\left\langle B_{p_{1}}\right\rangle$ vanish when $k=y$ and $\left\langle A_{v_{1}}\right\rangle$ vanishes and $\left\langle B_{p_{1}}\right\rangle \rightarrow+1$ when $k=z$.

The transition seen in $\left\langle A_{v_{1}}\right\rangle$ for the ground states of $H_{e}+$ $H_{\lambda}^{z}$ in Fig. 5(a) is consistent with $\lambda_{c} \simeq 0.33$. Both the transition point and the width of the transition region, which is due to finite-size effects, are comparable to the same quantities obtained from the magnetic order parameter. The anyons predict the transition point more accurately than the energy gap closing, which, for the same system size, happens only at $\lambda \simeq 0.7$.

$\left\langle B_{p_{1}}\right\rangle$ is not suitable for detecting the transition because $B_{p_{1}}$ commutes with all terms in $H_{m}+H_{\lambda}^{z}$. All energy eigenstates are therefore also eigenstates of $B_{p_{1}}$ with eigenvalue \pm 1 . As a result, $\left\langle B_{p_{1}}\right\rangle$ only measures whether the ground states have $B_{p_{1}}$ eigenvalue +1 or -1 . The first transition to a ground state with $B_{p_{1}}$ eigenvalue +1 happens around $\lambda \simeq 2.08$, but this does not exclude gap closings at smaller $\lambda$ values. These problems do not occur for $\left\langle A_{v_{1}}\right\rangle$, since $A_{v_{1}}$ does not commute with $H_{e}+H_{\lambda}^{z}$.

The transition seen in $\left\langle A_{v_{1}}\right\rangle\left(\left\langle B_{p_{1}}\right\rangle\right)$ in Fig. 5(b) for the ground states of $H_{e}+H_{\lambda}^{y}\left(H_{m}+H_{\lambda}^{y}\right)$ is consistent with $\lambda_{c}=$ 1 , and the transition is sharper than for the magnetic order parameter. The anyons also better predict the transition point than the energy gap closing, which happens around $\lambda \simeq 1.2$ for the same system size.

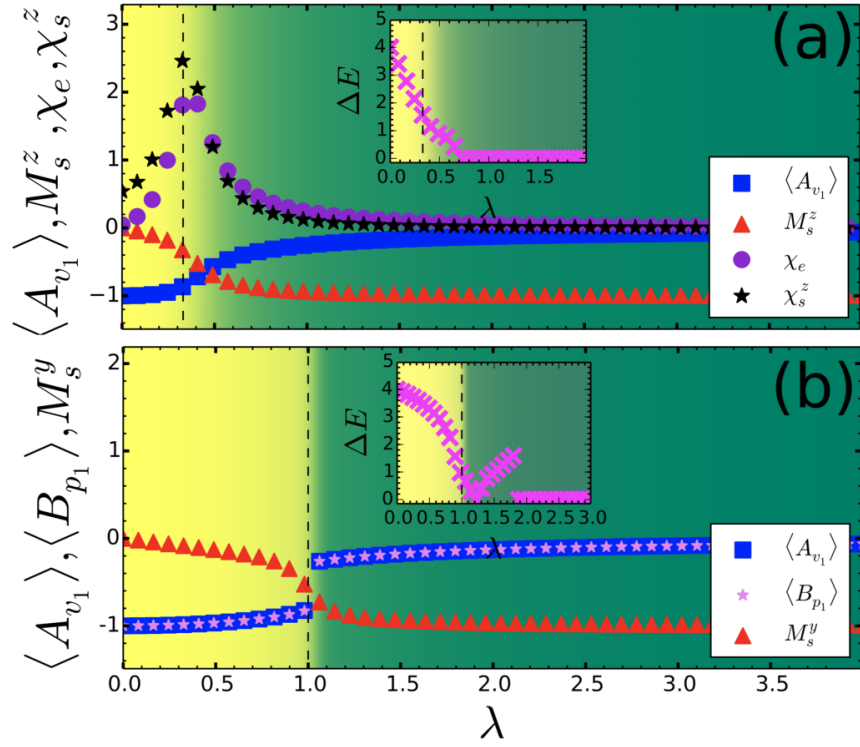

FIG. 5. (a) The toric code with a magnetic field of strength $\lambda$ in the $z$ direction undergoes a phase transition at $\lambda_{c} \simeq 0.33$. The transition is seen in $\left\langle A_{v_{1}}\right\rangle$, which detects the anyons in the ground states of $H_{e}+H_{\lambda}^{z}$, and in $M_{s}^{z}=\frac{1}{N}\left\langle\sum_{i} \sigma_{i}^{k}\right\rangle$, which is the magnetization per spin for the ground states of $H_{\mathrm{TC}}+H_{\lambda}^{z}$. We also show $\chi_{e}=$ $\partial\left\langle A_{v_{1}}\right\rangle / \partial \lambda$ and $\chi_{s}^{z}=-\partial M_{s}^{z} / \partial \lambda$. (b) When the magnetic field is in the $y$ direction, the phase transition happens at $\lambda_{c}=1$. We plot $\left\langle A_{v_{1}}\right\rangle$ for $H_{e}+H_{\lambda}^{y}$ and $\left\langle B_{p_{1}}\right\rangle$ for $H_{m}+H_{\lambda}^{y}$ as well as the magnetization per spin $M_{s}^{y}=\frac{1}{N}\left\langle\sum_{i} \sigma_{i}^{y}\right\rangle$ for $H_{\mathrm{TC}}+H_{\lambda}^{y}$. The closing of the energy gap $\Delta E$ between the ground state (fourfold degenerate) and the first excited state of $H_{\mathrm{TC}}+H_{\lambda}^{k}$ shown in the insets does not accurately predict the transition points. We use a $3 \times 3$ lattice with 18 spins in all cases, and the color gradient from yellow (topological phase) to green (nontopological phase) is plotted according to the value of $\left\langle A_{v_{1}}\right\rangle$.

\section{CONCLUSIONS}

We have shown that the properties of quasiparticles are an interesting tool to detect topological quantum phase transitions. The approach is to trap anyons in the ground state and study how their properties change, when the system crosses a phase transition. If we are able to create quasiparticles with robust and nontrivial braiding properties in a system, we know that the system hosts anyons of the observed type. For several quite different examples, we have demonstrated, however, that the phase transitions can be detected by observing a simple property, such as the charge of the anyons. This means that the phase transition points can be computed at low numerical costs.

The approach suggested here to detect topological quantum phase transitions is particularly direct, since, to fully exploit the interesting properties of topologically ordered systems, one needs to be able to create anyons in the systems and detect their properties. In the interacting Hofstadter model, the anyons can be created by adding a local potential, and the charge of the anyons used to detect the phase transition can be measured by measuring the expectation value of the number of particles on each site. Both of these can be done in experiments with ultracold atoms in optical lattices $[32,40]$. For the toric code model, the complexity of generating the 
Hamiltonians including the terms creating the anyons is about the same as generating the Hamiltonian without these terms, and the first steps towards realizing the Hamiltonian in experiments have been taken [41-43].

The ideas presented in this work can be applied as long as the two phases support quasiparticles with different properties and one can find suitable ways to create the quasiparticles. It is well suited for detecting phase transitions between different topologically ordered phases. In addition, it would be interesting to investigate what we can learn about transitions between nontopological phases by studying quasiparticles.

\section{ACKNOWLEDGMENTS}

We thank J. Vidal for helpful comments regarding previous work on phase transitions in the toric code in a magnetic field. J.W. acknowledges NSF Grant No. DMR 1306897 and NSF Grant No. DMR 1056536 for partial support.
[1] S. Sachdev, Quantum Phase Transitions (Cambridge University Press, Cambridge, UK, 2011).

[2] X.-G. Wen, Colloquium: Zoo of quantum-topological phases of matter, Rev. Mod. Phys. 89, 041004 (2017).

[3] A. G. Grushin, J. Motruk, M. P. Zaletel, and F. Pollmann, Characterization and stability of a fermionic $v=1 / 3$ fractional Chern insulator, Phys. Rev. B 91, 035136 (2015).

[4] J. Wildeboer, A. Seidel, and R. G. Melko, Entanglement entropy and topological order in resonating valence-bond quantum spin liquids, Phys. Rev. B 95, 100402(R) (2017).

[5] X. G. Wen and Q. Niu, Ground-state degeneracy of the fractional quantum Hall states in the presence of a random potential and on high-genus Riemann surfaces, Phys. Rev. B 41, 9377 (1990).

[6] R. Tao and F. D. M. Haldane, Impurity effect, degeneracy, and topological invariant in the quantum Hall effect, Phys. Rev. B 33, 3844 (1986).

[7] Q. Niu, D. J. Thouless, and Y.-S. Wu, Quantized Hall conductance as a topological invariant, Phys. Rev. B 31, 3372 (1985).

[8] K. Kudo, H. Watanabe, T. Kariyado, and Y. Hatsugai, ManyBody Chern Number without Integration, Phys. Rev. Lett. 122, 146601 (2019).

[9] T. Neupert, L. Santos, C. Chamon, and C. Mudry, Fractional Quantum Hall States at Zero Magnetic Field, Phys. Rev. Lett. 106, 236804 (2011).

[10] W.-J. Hu, S.-S. Gong, W. Zhu, and D. N. Sheng, Competing spin-liquid states in the spin- $\frac{1}{2}$ Heisenberg model on the triangular lattice, Phys. Rev. B 92, 140403(R) (2015).

[11] N. Regnault and B. A. Bernevig, Fractional Chern Insulator, Phys. Rev. X 1, 021014 (2011).

[12] R. Thomale, A. Sterdyniak, N. Regnault, and B. A. Bernevig, Entanglement Gap and a New Principle of Adiabatic Continuity, Phys. Rev. Lett. 104, 180502 (2010).

[13] M. Hermanns, A. Chandran, N. Regnault, and B. A. Bernevig, Haldane statistics in the finite-size entanglement spectra of $1 / \mathrm{m}$ fractional quantum Hall states, Phys. Rev. B 84, 121309(R) (2011).

[14] A. Sterdyniak, N. Regnault, and B. A. Bernevig, Extracting Excitations from Model State Entanglement, Phys. Rev. Lett. 106, 100405 (2011).

[15] M. Levin and X.-G. Wen, Detecting Topological Order in a Ground State Wave Function, Phys. Rev. Lett. 96, 110405 (2006).

[16] A. Kitaev and J. Preskill, Topological Entanglement Entropy, Phys. Rev. Lett. 96, 110404 (2006).

[17] H.-C. Jiang, Z. Wang, and L. Balents, Identifying topological order by entanglement entropy, Nat. Phys. 8, 902 (2012).
[18] P. Zanardi and N. Paunković, Ground state overlap and quantum phase transitions, Phys. Rev. E 74, 031123 (2006).

[19] D. Arovas, J. R. Schrieffer, and F. Wilczek, Fractional Statistics and the Quantum Hall Effect, Phys. Rev. Lett. 53, 722 (1984).

[20] P. Bonderson, V. Gurarie, and C. Nayak, Plasma analogy and non-Abelian statistics for Ising-type quantum Hall states, Phys. Rev. B 83, 075303 (2011).

[21] Y.-L. Wu, B. Estienne, N. Regnault, and B. A. Bernevig, Braiding Non-Abelian Quasiholes in Fractional Quantum Hall States, Phys. Rev. Lett. 113, 116801 (2014).

[22] S. Manna, J. Wildeboer, and A. E. B. Nielsen, Quasielectrons in lattice Moore-Read models, Phys. Rev. B 99, 045147 (2019).

[23] E. Macaluso, T. Comparin, L. Mazza, and I. Carusotto, Fusion Channels of non-Abelian Anyons from Angular-Momentum and Density-Profile Measurements, Phys. Rev. Lett. 123, 266801 (2019).

[24] M. Storni and R. H. Morf, Localized quasiholes and the Majorana fermion in fractional quantum hall state at $v=\frac{5}{2}$ via direct diagonalization, Phys. Rev. B 83, 195306 (2011).

[25] G. Moore and N. Read, Nonabelions in the fractional quantum Hall effect, Nucl. Phys. B 360, 362 (1991).

[26] I. Glasser, J. I. Cirac, G. Sierra, and A. E. B. Nielsen, Exact parent Hamiltonians of bosonic and fermionic Moore-Read states on lattices and local models, New J. Phys. 17, 082001 (2015).

[27] S. Manna, B. Pal, W. Wang, and A. E. B. Nielsen, Anyons and fractional quantum Hall effect in fractal dimensions, Phys. Rev. Research 2, 023401 (2020).

[28] M. Hafezi, A. S. Sørensen, E. Demler, and M. D. Lukin, Fractional quantum Hall effect in optical lattices, Phys. Rev. A 76, 023613 (2007).

[29] A. S. Sørensen, E. Demler, and M. D. Lukin, Fractional Quantum Hall States of Atoms in Optical Lattices, Phys. Rev. Lett. 94, 086803 (2005).

[30] E. Kapit, P. Ginsparg, and E. Mueller, Non-Abelian Braiding of Lattice Bosons, Phys. Rev. Lett. 108, 066802 (2012).

[31] A. E. B. Nielsen, I. Glasser, and I. D. Rodríguez, Quasielectrons as inverse quasiholes in lattice fractional quantum Hall models, New J. Phys. 20, 033029 (2018).

[32] M. Raciunas, F. N. Ünal, E. Anisimovas, and A. Eckardt, Creating, probing, and manipulating fractionally charged excitations of fractional Chern insulators in optical lattices, Phys. Rev. A 98, 063621 (2018).

[33] A. Kitaev, Anyons in an exactly solved model and beyond, Ann. Phys. 321, 2 (2006). 
[34] A. Kitaev, Fault-tolerant quantum computation by anyons, Ann. Phys. 303, 2 (2003).

[35] J. Vidal, S. Dusuel, and K. P. Schmidt, Low-energy effective theory of the toric code model in a parallel magnetic field, Phys. Rev. B 79, 033109 (2009).

[36] J. Vidal, R. Thomale, K. P. Schmidt, and S. Dusuel, Self-duality and bound states of the toric code model in a transverse field, Phys. Rev. B 80, 081104(R) (2009).

[37] S. Dusuel, M. Kamfor, R. Orús, K. P. Schmidt, and J. Vidal, Robustness of a Perturbed Topological Phase, Phys. Rev. Lett. 106, 107203 (2011).

[38] M. H. Zarei, Ising order parameter and topological phase transitions: Toric code in a uniform magnetic field, Phys. Rev. B 100, 125159 (2019).

[39] E. Greplova, A. Valenti, G. Boschung, F. Schäfer, N. Lörch, and S. D. Huber, Unsupervised identification of topological phase transitions using predictive models, New J. Phys. 22, 045003 (2020).

[40] C. Weitenberg, M. Endres, J. F. Sherson, M. Cheneau, P. Schauß, T. Fukuhara, I. Bloch, and S. Kuhr, Single-spin addressing in an atomic Mott insulator, Nature (London) 471, 319 (2011).

[41] T. Chen, S. Zhang, Y. Zhang, Y. Liu, S.-P. Kou, H. Sun, and X. Zhang, Experimental observation of classical analogy of topological entanglement entropy, Nat. Commun. 10, 1557 (2019).

[42] Z. Luo, J. Li, Z. Li, L.-Y. Hung, Y. Wan, X. Peng, and J. Du, Experimentally probing topological order and its breakdown through modular matrices, Nat. Phys. 14, 160 (2018).

[43] J. K. Pachos, W. Wieczorek, C. Schmid, N. Kiesel, R. Pohlner, and $\mathrm{H}$. Weinfurter, Revealing anyonic features in a toric code quantum simulation, New J. Phys. 11, 083010 (2009). 